Graphs and Combinatorics 3, 45-53 (1987)

Graphs and

Combinatorics

(C) Springer-Verlag 1987

\title{
The Class Reconstruction Number of Maximal Planar Graphs
}

\author{
Frank Harary ${ }^{1}$ and Josef Lauri ${ }^{2}$ \\ 1 The University of Michigan, Ann Arbor, MI 48109, USA \\ 2 The University of Malta, Msida, Malta
}

\begin{abstract}
The reconstruction number $r n(G)$ of a graph $G$ was introduced by Harary and Plantholt as the smallest number of vertex-deleted subgraphs $G_{i}=G-v_{i}$ in the deck of $G$ which do not all appear in the deck of any other graph. For any graph theoretic property $\mathscr{P}$, Harary defined the $\mathscr{P}$ reconstruction number of a graph $G \in \mathscr{P}$ as the smallest number of the $G_{i}$ in the deck of $G$, which do not all appear in the deck of any other graph in $\mathscr{P}$. We now study the maximal planar graph reconstruction number $\mathscr{M r n}(G)$, proving that its value is either 1 or 2 and characterizing those with value 1.
\end{abstract}

\section{Preliminaries}

All graphs considered are finite, simple and undirected, that is, graphs in the standard graph theoretic terminology of [3], except that here we shall use the terms vertex and edge instead of point and line respectively. We require some definitions which are not found in [3].

For brevity, a $k$-vertex has degree $k$, and an ordinary vertex has degree at least 4. The number of $k$-vertices of a graph $G$ is denoted by $n_{k}(G)$ and, for a vertex $v$ in $G$, the number of $k$-vertices adjacent to $v$ in $G$ is $n_{k}(v ; G)$ or simply $n_{k}(v)$.

A cycle $C_{k}$ on $k$ vertices is said to be a $k$-cycle. A face of a plane graph bounded by a $k$-cycle is a $k$-face. A representation in the plane of a planar graph is a $k$-representation if all the faces except one are (bounded by) 3-cycles and the exceptional face is a $k$-cycle, for some $k \geq 4$.

\section{The Reconstruction of Maximal Planar Graphs}

Since we shall be making extensive use of concepts and results obtained in [6], where the reconstructibility of maximal planar graphs was demonstrated, we present in this section a brief outline of how maximal planar graphs were reconstructed. In particular, we include here, for completeness sake, Theorems 2.2 and 2.3 of [6]. Full details of the ideas involved can be found in [6].

As in [6] we follow [7] and say that two plane representations $R$ and $R^{\prime}$ of a planar graph $G$ are equivalent if there exists an automorphism $\phi$ of $G$ such that $C$ is a boundary cycle of a face in $R$ iff $\phi(C)$ is a boundary cycle of a face in $R^{\prime}$. 
A maximal planar graph $G$ is degenerate if, for every ordinary vertex $v$ of $G$, the graph $G-v$ has at least two nonequivalent $\operatorname{deg}(v)$-representations. An example of a degenerate graph is given in Fig. 2.3 of [6].

Let $v$ be an ordinary vertex of a maximal planar graph $G$ and let $R$ be that $\operatorname{deg}(v)$-representation of $G-v$ in which all the neighbours of $v$ appear on the $\operatorname{deg}(v)$-face in a cycle order $v_{0}, v_{1}, \ldots, v_{\operatorname{deg}(v)+1}$ where $v_{i}$ is adjacent to $v_{i+1}$ (modulo $\operatorname{deg}(v))$. If $v_{i}$ is adjacent to $v_{i+2}$ and $\operatorname{deg}\left(v_{i+1}\right)>3$ (that is, the 3-cycle $T=v_{i} v_{i+1} v_{i+2} v_{i}$ is a separating triangle in $G$ ), then the subgraph induced by $G$ minus the vertices on the same side of $T$ as $v$ is called a span of $G$ incident to $v$. We denote this span by $S\left(v_{i} v_{i+1} v_{i+2}\right)$, and we call the vertices $v_{i}, v_{i+1}, v_{i+2}$ the primary vertices of $S$. The vertices $v_{i}$ and $v_{i+2}$ in particular are called the pivot vertices of the span and $v_{i+1}$ the replaced vertex. In any plane representation of the span, the edge $v_{i} v_{i+2}$ is incident to the faces bounded by the triangles $v_{i} v_{i+1} v_{i+2} v_{i}$ and $v_{i} y v_{i+2} v_{i}$ for a unique vertex $y \neq v_{i+1}$. This $y$ is called the replacement vertex of the span.

In [6] the following result was obtained. It is illustrated in Fig. 1.

Theorem A. Any other $\operatorname{deg}(v)$-representation $R^{\prime}$ of $G-v$ can be obtained from $R$ by a sequence of transformations in which replacement vertices replace corresponding replaced vertices on the $\operatorname{deg}(v)$-face.
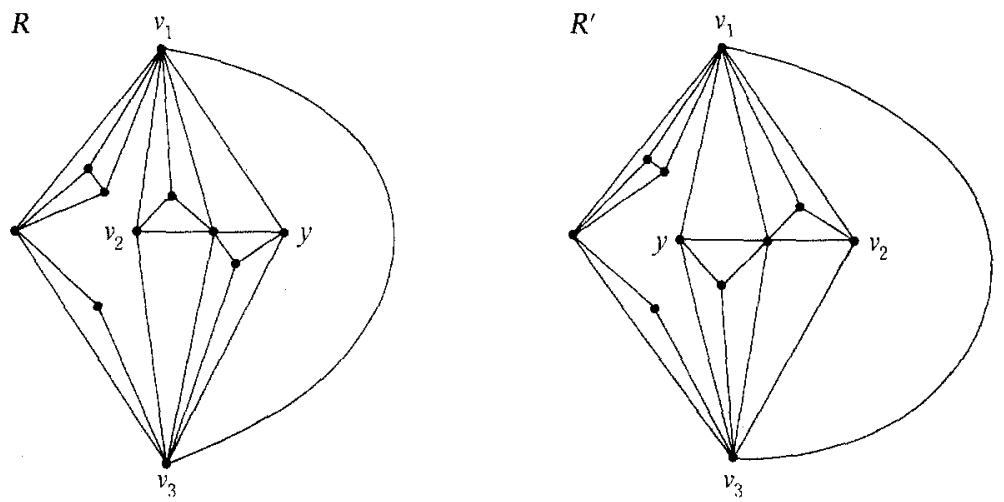

Fig. 1

This result is very important for reconstruction purposes since, reconstructing a maximal planar graph from $G-v$ amounts to taking a $\operatorname{deg}(v)$-representation $R$ of $G$, adding a new vertex, and joining it to all the vertices on the $\operatorname{deg}(v)$-face of $R$. The uniqueness or otherwise of the reconstructibility of $G$ hence depends on the uniqueness of the $\operatorname{deg}(v)$-representation of $G$, and this in turn, by Theorem $\mathrm{A}$, depends on the spans incident to $v$ in $G$. These considerations motivate our next definition.

Let $S$ be a maximal planar graph having three vertices labelled $a, b$ and $c$ on a face. Let $G$ be a maximal planar graph having an ordinary vertex $v$ incident to a span $S(a b c)$ isomorphic to $S$; and let $y$ be the replacement vertex of this span. Let $R$ denote that $\operatorname{deg}(v)$-representation of $G-v$ in which all the neighbours of $v$ appear on the $\operatorname{deg}(v)$-face, and let $R^{\prime}$ be that $\operatorname{deg}(v)$-representation of $G-v$ obtained from 

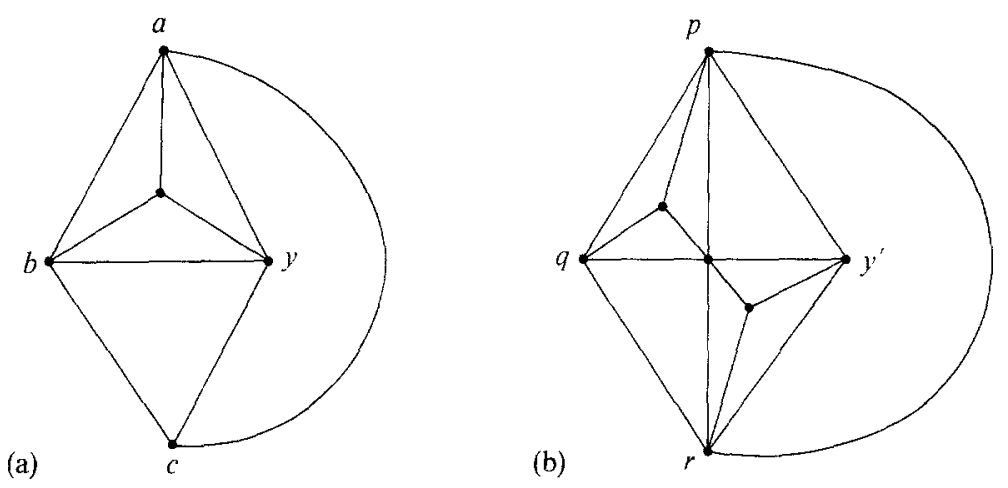

Fig. 2

$R$ by replacing the vertex $b$ by the vertex $y$ on the $\operatorname{deg}(v)$-face. If there is some such $G$ for which the embeddings $R$ and $R^{\prime}$ are not equivalent, then we say that the span $S(a b c)$ is asymmetric. A span which is not asymmetric is said to be symmetric (on pivot vertices $a$ and $c$ ). Thus, for example, the span $S(a b c)$ in Fig. 2(a) is symmetric, whereas the span $S(p q r)$ in Fig. 2(b) is asymmetric.

Note that if $G$ is degenerate then every ordinary vertex of $G$ is incident to at least one asymmetric span. If $v$ is an ordinary vertex of $G$ such that $v$ is incident to exactly one asymmetric span $S(a b c)$, with replacement vertex $y$, and moreover $\operatorname{deg}(b) \neq \operatorname{deg}(y)+1$, we then say that $v$ is a good vertex of $G$. We now give Theorems $B$ and $C$ which are respectively Theorems 2.2 and 2.3 of [6]. In fact, Theorem $B$ is an improved version of Theorem 2.2, since here only three types of graph have to be considered (these are Types I, II with $k=2$, and $V$ of Theorem B 2.2 in [6]). It can easily be seen that this version follows from Theorem 2.2 since if $G$ contains an ordinary vertex incident to any of the other types of span given in Theorem 2.2, then it must contain an ordinary vertex incident to one of types of spans specified in Theorem B.

Theorem B. Let $G$ be a degenerate graph. Then there exists an ordinary vertex $u$ in $G$ such that $u$ is incident to an asymmetric span $S$, with $a, b, c$ as primary vertices, $S$ being one of the three types of graphs shown below.

Theorem C. Every degenerate graph has a good vertex.

Proof. We outline the proof in [6]. In particular we list the possible types of good vertices which can arise, since these will be required later.

Let $G$ be a degenerate graph. Therefore by Theorem B there exists an ordinary vertex $u$ incident to an asymmetric span $S$, this span being one of the three types of graph shown in Fig. 3.

We have to consider these three cases, taking into consideration the different possibilities which arise depending on which two of $a, b, c$ are the pivot vertices of $S$ considered as an asymmetric span incident to $u$.

In this way we obtain Table 1 which gives, in each case, the good vertex $x$, and the degrees of the replaced and replacement vertex of the asymmetric span incident to $x$. 

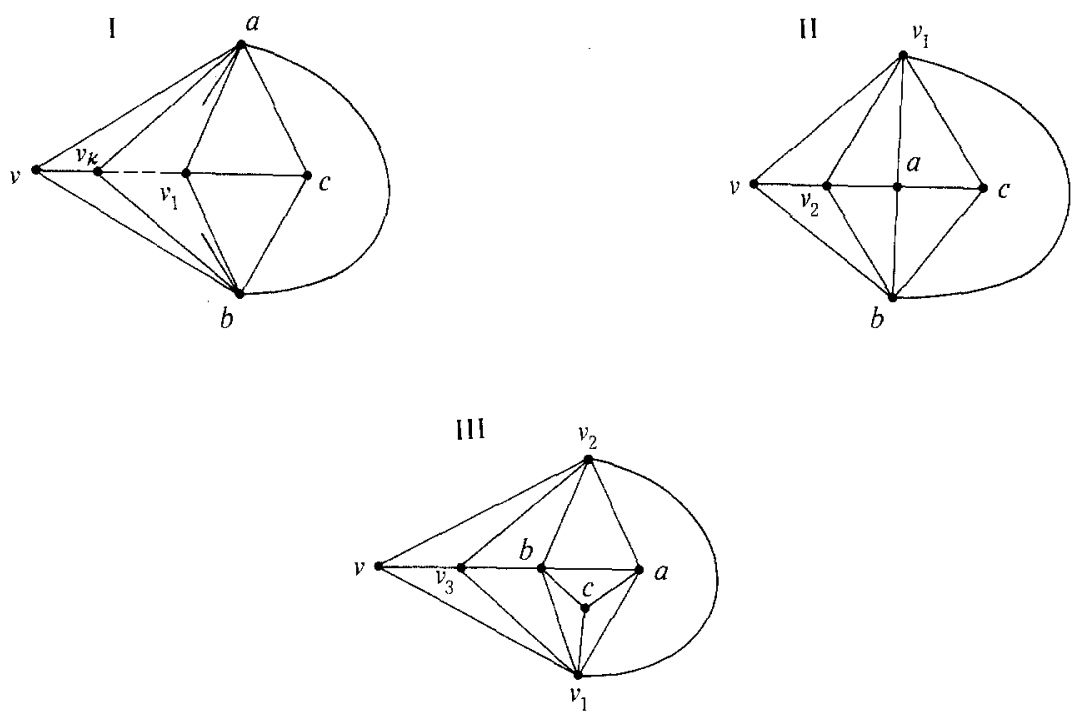

Fig. 3

Table 1

\begin{tabular}{|c|c|c|c|c|c|}
\hline $\begin{array}{l}\text { Type } \\
\text { of } S\end{array}$ & $\begin{array}{l}\text { Pivot } \\
\text { vertices } \\
\text { of } S\end{array}$ & $\begin{array}{l}\text { Good } \\
\text { vertex } \\
x\end{array}$ & $\begin{array}{l}\text { Asymmetric } \\
\text { span } S_{x} \\
\text { incident } \\
\text { to } x\end{array}$ & $\begin{array}{l}\text { Degree } \\
\text { in } G \text { of } \\
\text { replaced } \\
\text { vertex } \\
\text { of } S_{x}\end{array}$ & $\begin{array}{l}\text { Replacement } \\
\text { vertex of } S_{x} \\
\text { and its } \\
\text { degree in } G\end{array}$ \\
\hline I & $\begin{array}{l}a \text { and } c \\
\text { or } b \& c\end{array}$ & $v_{k}$ & $S_{x}\left(b v_{k-1} a\right)$ & 4 & $u ; \operatorname{deg}(u)>3$ \\
\hline $\begin{array}{l}\text { II } \\
\text { (i) } \\
\text { (ii) }\end{array}$ & $\begin{array}{l}a \text { and } b \\
b \text { and } c\end{array}$ & $\begin{array}{l}v_{2} \\
v_{2}\end{array}$ & $\begin{array}{l}S_{x}\left(v_{1} a b\right) \\
S_{x}\left(v_{1} a b\right)\end{array}$ & $\begin{array}{l}>5 \\
5\end{array}$ & $\begin{array}{l}c ; \operatorname{deg}(c)=4 \\
c ; \operatorname{deg}(c)>4\end{array}$ \\
\hline $\begin{array}{l}\text { III } \\
\text { (i) } \\
\text { (ii) } \\
\text { (iii) }\end{array}$ & $\begin{array}{l}a \text { and } b \\
c \text { and } b \\
c \text { and } a\end{array}$ & $\begin{array}{l}v_{2} \\
v_{2} \\
v_{3}\end{array}$ & $\begin{array}{l}S_{x}\left(v_{1} a b\right) \\
S_{x}\left(v_{1} a b\right) \\
S_{x}\left(v_{1} b v_{2}\right)\end{array}$ & $\begin{array}{l}>5 \\
5 \\
6\end{array}$ & $\begin{array}{l}c ; \operatorname{deg}(c)=4 \\
c ; \operatorname{deg}(c)>4 \\
a ; \operatorname{deg}(a)>5\end{array}$ \\
\hline
\end{tabular}

Using Theorem $\mathrm{C}$ the following result was then obtained in [6].

Theorem D. Every maximal planar graph is reconstructible.

Proof. The argument bricfly runs as follows. It was first shown, in [2], that maximal planar graphs are recognizable, that is, if $G$ is a maximal planar graph, then any other graph which is reconstructible from the deck of vertex-deleted subgraphs of $G$ must also be maximal planar. It then follows that we need only consider maximal planar graphs which are degenerate because, if $G$ is not degenerate, then there is an 
ordinary vertex $v$ such that $G-v$ has a unique (up to plane equivalence) $\operatorname{deg}(v)$ representation. But then $G$ is uniquely reconstructible from $G-v$ by adding a new vertex and joining it to all the vertices on the $\operatorname{deg}(v)$-face of the $\operatorname{deg}(v)$-representation of $G$.

Assuming therefore that $G$ is degenerate it follows, by Theorem $C$, that $G$ has a good vertex $x$, incident to a unique asymmetric span with replaced and replacement vertices $r$ and $r^{\prime}$ respectively, say. Hence, in this case, there are two ways to reconstruct a maximal planar graph from $G-x$, either as $G$ itself or as $H=$ $G-x r+x r^{\prime}$. But from the definition of a good vertex it follows that the degree sequences of $G$ and $H$ are different. However, from the vertex-deck of $G$ the degree sequence of $G$ can be determined, and therefore $G$ can be distinguished as the only possible reconstruction from $G-x$.

\section{The Class Reconstruction Number}

Class reconstruction numbers were first introduced in [4]. We shall here study the class reconstruction number $\mathscr{M} r n(G)$ where $\mathscr{M}$ is the class of maximal planar graphs. The problem therefore is this. We are given the information that $G$ is maximal planar and we are required to find $\mathscr{M} n(G)$, that is, the smallest number of vertex-deleted subgraphs in the deck of $G$ which are not all in the deck of any other maximal planar graph. Therefore here, unlike the previous section, we do not have the degree sequence of $G$, and the above method of reconstructing from just one $G-v$, with $v$ a good vertex, does not work.

We first observe that $\mathscr{M} r n(G)=1$ if $G$ is not degenerate, because in this case there is some $G-v, v$ an ordinary vertex with a unique $\operatorname{deg}(v)$-representation, so that knowing that $G$ is maximal planar we can reconstruct it uniquely from this $\operatorname{deg}(v)$-representation, as described above. In particular this means that if the minimum degree in $G$ is at least 4 , then $\mathscr{M} r n(G)=1$, since a degenerate graph necessarily has minimum degree 3. (This last assertion follows from the following considerations. Every maximal planar graph is 3-connected and therefore, by a result in [1], a maximal planar graph $G$ with minimum degree at least 4 has a vertex $v$ such that $G-v$ is 3 -connected. But then, by a result in [8], $G-v$ has a unique plane representation, showing that $G$ cannot be degenerate.)

As we have seen, the idea of a good vertex was of central importance in the reconstruction of maximal planar graphs. We now need to extend this idea somewhat. An ordinary vertex $v$ of $G$ is said to be a useful vertex if $v$ is incident to exactly one asymmetric span in $G$. Note that we do not make any restrictions on the degrees of the replaced and replacement vertices of the asymmetric span, and that any good vertex is a useful vertex. We now develop a sequence of four lemmas to prove the main result.

Lemma 1. Let $G$ be a degenerate graph and $x$ a useful vertex of $G$ incident to the asymmetric span $S(a b c)$ with replacement vertex $y$. Let $H=G-x b+x y$. If $\mathscr{M r n}(G)>2$, then for any $z$ in $V(G)$ there is some vertex $z^{\prime}$ in $V(H)$ such that $G-z$ is isomorphic to $H-z^{\prime}$.

Proof. The only possible maximal planar reconstructions from $G-x$ are $G$ itself or $H$ (that is, $G$ and $H$ are the only maximal planar graphs which can have in their 
deck a subgraph isomorphic to $G-x$ ). Now, since $\mathscr{M} r n(G)>2$, it follows that for any $z$ in $V(G), z \neq x$, either $G-z$ is isomorphic to $G-x$, in which case the required result follows since $G-x=H-x$, or else there must be some maximal planar graph $J$, not isomorphic to $G$, such that the vertex-deck of $J$ contains both a graph isomorphic to $G-x$ and one isomorphic to $G-z$. But since there are only two ways to reconstruct a maximal planar graph from $G-x$, that is either as $G$ itself or as $H$, then $J$ is isomorphic to $H$, and therefore the vertex-deck of $H$ contains a subgraph isomorphic to $G-z$, as required.

Lemma 2. Let $x$ be a good vertex of a degenerate graph $G$ incident to the asymmetric span $S(a b c)$ with replacement vertex $y$, and let $x^{\prime}$ be a useful vertex of $G$, incident to the asymmetric span $S\left(a^{\prime} b^{\prime} c^{\prime}\right)$, with replacement vertex $y^{\prime}$. Let $H=G-x b+x y$ and $H^{\prime}=G-x^{\prime} b^{\prime}+x^{\prime} y^{\prime}$. If $\operatorname{deg}(b) \neq \operatorname{deg}\left(b^{\prime}\right)$, then the degree sequence of $H$ is different from that of $H^{\prime}$.

Proof. Since $x$ is a good vertex then $\operatorname{deg}(b) \neq \operatorname{deg}(y)+1$. It therefore follows that, if $s=\operatorname{deg}(b)$, then $n_{s}(H)<n_{s}(G)$, and $n_{s-1}(H)>n_{s-1}(G)$. Now, let $s^{\prime}=\operatorname{deg}\left(b^{\prime}\right)$ and $t^{\prime}=\operatorname{deg}\left(y^{\prime}\right)$. We observe first that if $s^{\prime}=t^{\prime}+1$, then the degree sequence of $H^{\prime}$ is the same as that of $G$, and therefore different from that of $H$. We may therefore assume that $s^{\prime} \neq t^{\prime}+1$, that is, $x^{\prime}$ is a good vertex. Now, the only degrees in the degree sequences of $G$ and $H^{\prime}$ which differ are $s^{\prime}, s^{\prime}-1, t^{\prime}, t^{\prime}+1$. In fact, since $s^{\prime} \neq t^{\prime}+1$, then

$$
n_{s^{\prime}-1}(H)>n_{s^{\prime}-1}(G) \text { and } n_{s^{\prime}}(H)<n_{s^{\prime}}(G)
$$

and $n_{t^{\prime}}\left(H^{\prime}\right)<n_{t^{\prime}}\left(G^{\prime}\right)$ and $n_{t^{\prime}+1}\left(H^{\prime}\right)>n_{t^{\prime}+1}\left(G^{\prime}\right)$. But $n_{s-1}(H)>n_{s-1}(G)$ and $n_{s}(H)<$ $n_{s}(G)$, and therefore the only way that the degree sequence of $H$ and $H^{\prime}$ can be the same is if $s=s^{\prime}$.

Lemma 3. Let $G$ be a degenerate graph and assume that $x$ is a good vertex of $G$ and $x^{\prime}$ a useful vertex of $G$. Let $x$ and $x^{\prime}$ be incident to the asymmetric spans $S$ and $S^{\prime}$ respectively, and let the degrees of the replaced vertices of $S$ and $S^{\prime}$ be $s$ and $s^{\prime}$ respectively. If $s \neq s^{\prime}$, then $\mathscr{M} r n(G)=2$.

Proof. Let $S=S(a b c)$ and let $y$ be the replacement vertex of $S$. Therefore $s \neq$ $\operatorname{deg}(y)+1$. Let $H=G-x b+x y$. Also, let $S^{\prime}=S^{\prime}\left(a^{\prime} b^{\prime} c^{\prime}\right)$ and let $y^{\prime}$ be the replacement vertex of $S^{\prime}$ and $H^{\prime}=G-x^{\prime} b^{\prime}+x^{\prime} y^{\prime}$.

If $s \neq s^{\prime}$, then by Lemma 2 the degree sequences of $H$ and $H^{\prime}$ are different, and therefore $H$ and $H^{\prime}$ are not isomorphic.

But by Lemma 1, if $\mathscr{M} r n(G)>2$, then the graph $G-x^{\prime}$ must be isomorphic to some graph in the deck of $H$. However, the only possible maximal planar reconstructions from $G-x^{\prime}$ are $G$ or $H^{\prime}$ (that is, $G$ and $H^{\prime}$ are the only maximal planar graphs which can have in their deck a subgraph isomorphic to $G-x^{\prime}$ ). This is however impossible since $H$ is not isomorphic to $G$ (because $x$ is a good vertex of $G)$, and $H$ is not isomorphic to $H^{\prime}\left(\right.$ since $\left.s \neq s^{\prime}\right)$.

Lemma 4. Let $G$ and $H$ be two graphs such that for $s \geq 2, n_{s-1}(G)<n_{s-1}(H)$ and $n_{k}(G)=n_{k}(H)$ for all $0 \leq k<s-1$. Assume that the vertices $z$ in $V(G)$ and $z^{\prime}$ in $V(H)$ are such that $\operatorname{deg}(z)=\operatorname{deg}\left(z^{\prime}\right)$ and $n_{k}(G-z)=n_{k}\left(H-z^{\prime}\right)$ for all $0 \leq k \leq s-1$. Then $n_{k}(z ; G)=n_{k}\left(z^{\prime} ; H\right)$ for all $0 \leq k \leq s-1$ and $n_{s}(z ; G)>n_{s}\left(z^{\prime} ; H\right)$. 
Proof. (Note that we shall henceforth write $n_{k}(z)$ instead of $n_{k}(z ; G)$ etc., dropping the references to $G$ and $H$.) Let $p=\operatorname{deg}(z)=\operatorname{deg}\left(z^{\prime}\right)$, and let $\delta_{i j}$ be the Kronecker delta. We now observe that, for any $k \geq 0$,

$$
n_{k}(G-z)=n_{k}(G)+n_{k+1}(z)-n_{k}(z)-\delta_{k p}
$$

and

$$
n_{k}\left(H-z^{\prime}\right)=n_{k}(H)+n_{k+1}\left(z^{\prime}\right)-n_{k}\left(z^{\prime}\right)-\delta_{k p} .
$$

Therefore for $0 \leq k \leq s-1$,

$$
n_{k}(G)+n_{k+1}(z)-n_{k}(z)=n_{k}(H)+n_{k-1}\left(z^{\prime}\right)-n_{k}\left(z^{\prime}\right) .
$$

Since $n_{0}(z)=n_{0}\left(z^{\prime}\right)=0$, then for $k=0,(1)$ gives,

$$
n_{0}(G)+n_{1}(z)=n_{0}(H)+n_{1}\left(z^{\prime}\right) .
$$

But $n_{0}(G)=n_{0}(H)$, therefore $n_{1}^{\prime}(z)=n_{1}\left(z^{\prime}\right)$. Using (1) successively for $k=1,2, \ldots$, $s-2$ then gives,

$$
n_{k}(z)=n_{k}\left(z^{\prime}\right) \text { for all } 0 \leq k \leq s-1 .
$$

Then, for $k=s-1,(1)$ gives,

$$
\begin{aligned}
n_{s-1}(G)+n_{s}(z)-n_{s-1}(z) & =n_{s-1}(H)+n_{s}\left(z^{\prime}\right)-n_{s-1}\left(z^{\prime}\right) \\
& >n_{s-1}(G)+n_{s}\left(z^{\prime}\right)-n_{s-1}\left(z^{\prime}\right) .
\end{aligned}
$$

Therefore $n_{s}(z)>n_{s}\left(z^{\prime}\right)$ (since $\left.n_{s-1}(z)=n_{s-1}\left(z^{\prime}\right)\right)$.

Theorem 1. If $G$ is a degenerate graph, then $\mathscr{M} r n(G)=2$.

\begin{tabular}{|c|c|c|c|c|c|}
\hline $\begin{array}{l}\text { Type } \\
\text { of } S\end{array}$ & $\begin{array}{l}\text { Pivot } \\
\text { vertices } \\
\text { of } S\end{array}$ & $\begin{array}{l}\text { Useful } \\
\text { vertex } \\
x^{\prime}\end{array}$ & $\begin{array}{l}\text { Asymmetric } \\
\text { span } S_{x^{\prime}} \\
\text { incident } \\
\text { to } x^{\prime}\end{array}$ & $\begin{array}{l}\text { Degree } \\
\text { in } G \text { of } \\
\text { replaced } \\
\text { vertex } \\
\text { of } S_{x^{\prime}}\end{array}$ & $\begin{array}{l}\text { Degree } \\
\text { in } G \text { of } \\
\text { replaced } \\
\text { vertex } \\
\text { of } S_{x}\end{array}$ \\
\hline I & $\begin{array}{l}a \text { and } c \\
\text { or } b \& c\end{array}$ & $v_{1}$ & $S_{x^{\prime}}(a c b)$ & $>4$ & 4 \\
\hline $\begin{array}{l}\text { II } \\
\text { (i) }\end{array}$ & $a$ and $b$ & $v_{1}$ & $S_{x^{\prime}}(a c b)$ & 4 & $>5$ \\
\hline $\begin{array}{l}\text { III } \\
\text { (i) } \\
\text { (ii) }\end{array}$ & $\begin{array}{l}a \text { and } b \\
c \text { and } b\end{array}$ & $\begin{array}{l}v_{1} \\
v_{3}\end{array}$ & $\begin{array}{l}S_{x^{\prime}}(b c a) \\
S_{x^{\prime}}\left(v_{2} b v_{1}\right)\end{array}$ & $\begin{array}{l}4 \\
\geq 7\end{array}$ & $\geq 6$ \\
\hline
\end{tabular}

Proof. By Theorem C, $G$ has a good vertex $x$ in one of the configurations listed in Table 1. We shall consider all these cases and we shall show that for most of them we can find a useful vertex $x^{\prime}$ such that $x$ and $x^{\prime}$ satisfy the conditions of Lemma 3. This is done in Table 2 below. The remaining cases (II(ii) and III(iii)) are then treated separately using Lemma 4.

Table 2 


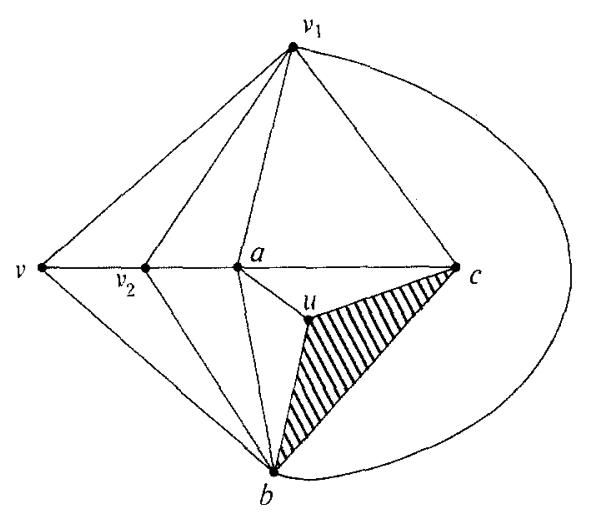

Fig. 4

In Table II, for each of the cases considered, we give the useful vertex $x^{\prime}$, the asymmetric span $S_{x^{\prime}}$ incident to $x^{\prime}$, and the degree of the replaced vertex of $S_{x^{\prime}}$. For comparison we also give (from Table I) the degree of the replaced vertex of the asymmetric span $S_{x}$ incident to $x$. In all the cases, the two degrees are different, showing by Lemma 3 that $\mathscr{M} r n(G)=2$ in these cases.

We are therefore left with cases II(ii) and III(iii). Let us consider first case II(ii), that is when the span $S$ of Theorem B is of type II and the pivot vertices of $S$ are $b$ and $c$ (see Fig. 4), when the vertex $v_{2}$ is a good vertex incident to the asymmetric span $S\left(v_{1} a b\right)$ with replacement vertex $c$, when the degree of $a$ in $G$ is 5 , and when the degree of $c$ in $G$ is at least 5 (see Fig. 4). Therefore letting $H=G-v_{2} a+v_{2} c$ we find that $n_{4}(H)>n_{4}(G)$ and $n_{k}(H)=n_{k}(G)$ for all $0 \leq k<4$.

We may assume that, in $G, c$ is not a 4-vertex or a 5-vertex adjacent to a 3-vertex. Therefore there is a one-one correspondence between the 3-vertices of $G$ and those of $H$ such that the corresponding 3-vertices have the same number of neighbours with degree 5 (since $a$ is not adjacent to any 3 -vertex and $a$ and $c$ are the only vertices which change degree). We therefore let $z$ be a 3-vertex of $G$ such that $z$ is adjacent to a minimal number of 5 -vertices in $G$. Therefore, by the previous remarks, for any

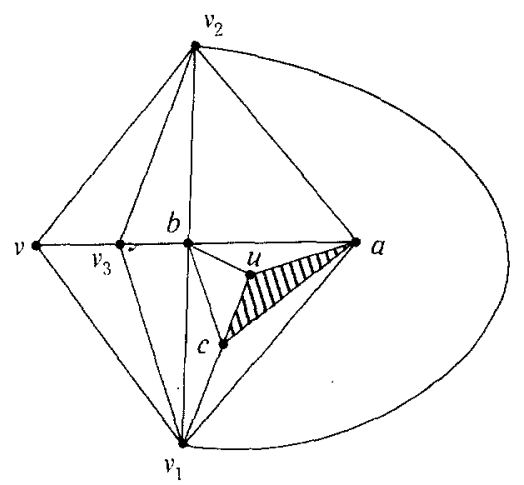

Fig. 5 
3-vertex $z^{\prime}$ in $H, n_{5}(z) \leq n_{5}\left(z^{\prime}\right)$. Hence, by Lemma 4, there can be no 3-vertex $z^{\prime}$ such that $G-z$ and $H-z^{\prime}$ are isomorphic, so by Lemma $1, \mathscr{M r n}(G)=2$.

We can now deal similarly with case III(iii), that is, when $S$ is of type III with $a$ and $c$ as pivots (Fig. 5).

The vertex $v_{3}$ is a good vertex incident to the asymmetric span $S\left(v_{2} b v_{1}\right)$ with replacement vertex $a$. The degree of $b$ is 6 , while that of $a$ is at least 6 . Let $H=G-v_{3} b+v_{3} a$. Therefore $n_{5}(H)>n_{5}(G)$ and $n_{k}(H)=n_{k}(G)$ for all $0 \leq k<5$.

Again, we may assume that, in $G, a$ is not a 5-vertex or a 6-vertex adjacent to a 3-vertex.

Hence, by considering a 3-vertex of $G$ adjacent to a minimum number of 6-vertices, we obtain as above that $\mathscr{M} r n(G)=2$.

\section{References}

1. Chartrand, G., Kaugars, A., and Lick, D.R.: Critically $n$-connected graphs. Proc. Amer. Math. Soc. 32, 63-68 (1972)

2. Fiorini, S., Lauri, J.: The reconstruction of maximal planar graphs. I. Recognition. J. Comb. Theory (B) 30, 188-195 (1981)

3. Harary, F.: Graph Theory. Reading: Addison-Wesley 1969

4. Harary, F.: Conditional graph theory: Colorability, connectivity, reconstruction. Proc. First China-USA Conf. on Graph Theory (to appear)

5. Harary, F., Plantholt, M.: The graph reconstruction number. J. Graph Theory 9, 451-454 (1985).

6. Lauri, J.: The reconstruction of maximal planar graphs. II. Reconstruction. J. Comb. Theory (B) 30, 196-214 (1981)

7. Ore, O.: The Four-Color Problem. New York: Academic Press 1967

8. Whitney, H.: Congruent graphs and the connectivity of graphs. Amer. J. Math. 54, 150-168 (1932)

Received: July 10, 1985

Revised: July 8, 1986 